\title{
Sex Differences in the Association Between Risk of Obstructive Sleep Apnea, Insomnia, and Frailty in Older Adults
}

\author{
Karla Moreno-Tamayo (D) \\ Betty Manrique-Espinoza (iD ${ }^{2}$ \\ Selene Guerrero-Zúñiga (iD ${ }^{3}$ \\ Eliseo Ramírez-García (iD) \\ Sergio Sánchez-García (ID) \\ 'Unidad de Investigación Epidemiológica \\ y en Servicios de Salud, Área \\ Envejecimiento, Centro Médico Nacional \\ Siglo XXI, Instituto Mexicano del Seguro \\ Social, Ciudad de México, México; \\ ${ }^{2}$ Centro de Investigación en Evaluación \\ y Encuestas, Instituto Nacional de Salud \\ Pública, Cuernavaca, Morelos, México; \\ ${ }^{3}$ Unidad de Medicina del Sueño, Instituto \\ Nacional de Enfermedades Respiratorias \\ Ismael Cosío Villegas, Ciudad de México, \\ México
}

Purpose: To analyze the association between risk of obstructive sleep apnea (OSA), insomnia, sleepiness and self-assessed sleep duration with frailty in older adults.

Patients and Methods: Cross-sectional study with 1643 older adults (60 to 97 years old) who participated in round 6 (2019) of the Cohort of Obesity Sarcopenia and Obesity in Older Adults of the Mexican Institute of Social Security. The Berlin Questionnaire was used to assess risk of OSA, the Athens Insomnia Scale for insomnia, the Epworth Scale for sleepiness, and sleep duration by self-report. Frailty was assessed with the frailty criteria proposed by Fried. Sociodemographic and health variables were also collected. Statistical analysis was performed with logistic regression and was stratified by sex.

Results: This study included 1643 participants ( 823 men and 820 women). Mean age was $67.1 \pm 5.9$ years. The percentage of frail older adults was $10.5 \%(\mathrm{n}=172)$, with risk of OSA $26.1 \%(n=429)$, with insomnia $30.3 \%(n=497)$ and with sleepiness $18.9 \%(n=310)$. In all participants, risk of OSA and insomnia were associated with frailty. In the stratified analysis by sex, the association between risk of OSA and frailty was observed in women (odds ratio $(\mathrm{OR})=1.84,95 \%$ confidence interval $(\mathrm{CI}) 1.05,3.22)$, but not in men $(\mathrm{OR}=1.19,95 \% \mathrm{CI}$ : $0.65,2.19)$. Insomnia was significantly associated with frailty in women (OR $=2.38,95 \%$ CI: $1.35,4.20)$ and in men (OR $=1.88,95 \%$ CI: 1.01, 3.52). Neither sleepiness nor sleep duration was associated with frailty.

Conclusion: In community-dwelling older adults, both the risk of OSA and insomnia conferred greater odds of presenting frailty in women. It is required to implement strategies aimed at improving sleep hygiene and detecting patients with OSA and insomnia.

Keywords: sleep disorders, obstructive sleep apnea, older adults, insomnia disorder, sleepiness

\section{Introduction}

Frailty is a geriatric syndrome in which the physiological reserve is diminished. It affects $10.7 \%$ of older adults in high-income countries ${ }^{1}$ and $17.4 \%$ in middleincome countries. ${ }^{2}$ Although frailty is strongly associated with adverse outcomes such as hospitalization and death, ${ }^{3,4}$ it can be reversible and preventable, which is why it has been important to identify its related factors. ${ }^{4}$ In recent years there has been a proliferation of research which focuses on the relationship between sleep and frailty. Sleep is a complex, dynamic and essential process for nocturnal restoration and wellbeing during the day; thus, disturbed sleep would affect the physiological reserve in older people through the disruption of hormonal pathways, immune
Correspondence: Sergio Sánchez-García Unidad de Investigación Epidemiológica y en Servicios de Salud, Área Envejecimiento, Centro Médico Nacional Siglo XXI, Instituto Mexicano del Seguro Social, Avenida Cuauhtémoc No. 330, Edificio CORCE, Tercer piso. Col. Doctores, Alcaldía Cuauhtémoc, Ciudad de México, 06720, México

Tel + 52556276900 Ext. 21846

Email sergio.sanchezga@imss.gob.mx 
dysfunction and chronic inflammation which contribute to the imbalance of muscle metabolism and homeostasis. ${ }^{5}$ Sleep disturbances not only may impair metabolism, but also result in fatigue during waking hours which may trigger napping throughout the day or prevent older people to be active that may contribute to perpetuate the worse sleep cycle and frailty.

Studies which have focused on analyzing sleep duration support the idea that insufficient sleep ( $<6$ hours) or more than the recommended time ( $>8$ hours) is associated with a greater possibility of frailty. ${ }^{6-9}$ It has been postulated that older people with daytime sleepiness, sleep disorders such as insomnia or with sleep-related breathing disorders, face greater chances of being frail. ${ }^{10}$ Other evidence has also shown that poor quality of sleep, sleep latency, sleep efficiency and sleep-related breathing disorders have implications on frailty. ${ }^{6,11-16}$

In addition, it has been observed that the modifying role of sex on the association between sleep and frailty might mainly occur among women older adults. Previous studies indicated that sleep complaint, sleep duration, and sleep-breathing disorders were only associated with frailty in older women but not in men. ${ }^{11,14,15}$ However, it is nonconclusive since men and women sleep differently. For instance, women tend to self-report less sleep duration; insomnia is more commonly reported by women than men, while the risk of obstructive sleep apnea (OSA) predominates in men. ${ }^{17}$ Results from observational studies comprised of men participants have shown that prolonged sleep latency, poor subjective sleep quality, and nocturnal hypoxemia were associated with frailty/or mortality. ${ }^{18,19}$ In light of these facts, it seems that the variability of the association between sleep and frailty regarding sex difference needs to be addressed.

It appears that previous research on the relationship between sleep and frailty has followed the pattern focusing on single concepts, especially based on sleep duration or sleep quality. ${ }^{7-9,12,15}$ Although these research has provided invaluable inputs, data on sleep should be balanced with distinct notion of sleep disturbances since this health problem requires a more detailed perspective to distinguish how a different sleep concept affects in the presence of the other one. For instance, a study reported that sleepiness was linked to increased frailty but insomnia was not. ${ }^{16}$ However, such study failed to clarify if the association between sleepiness and frailty remained after considering or at least screening for other sleep disorders, such as risk of OSA. Thus, based on current evidence and sex gap, the objective of this study was to analyze the association of risk of OSA, insomnia, sleepiness and self-assessed sleep duration with frailty globally, and stratified by sex.

\section{Patients and Methods Study Design and Participants}

Cross-sectional study with information from wave six of the "Cohort of Obesity, Sarcopenia and Frailty of Older Mexican Adults" (COSFOMA). COSFOMA procedures have been previously described. ${ }^{20}$ Briefly, this is a population-based prospective study that began in 2014 with the participation of 1252 adults aged 60 years or more who were beneficiaries of the Mexican Institute of Social Security (IMSS, by its Spanish acronym) in Mexico City. COSFOMA collected information through interviews and geriatric tests conducted by previously trained personnel at annual intervals until 2019. In total, COSFOMA has six survey rounds available, with wave six being the most recent. In this wave of COSFOMA, 486 (38.8\%) participants were still under study. In wave six, carried out from April to September 2019, the sample was refreshed with 1192 adults aged 60 years and older who were added to compensate for losses to follow-up and mortality, and following the same sampling strategy of $2014 .^{20}$ Briefly, the sampling frame was the $1,127,720$ older adults registered at the 48 primary health care called Family Medicine Units at IMSS Mexico City in 2018. Through a simple random selection procedure, 20,000 records were selected. To 6000 of the eligible records (ie, complete postal address) a letter was sent, inviting subjects to attend a comprehensive health evaluation at the IMSS. A total of 2385 received the invitation letter in which the nature of study participation was explained, with the field center telephone number provided. Telephone contact was made with 1375 older adults, but only 1192 (86.7\%) attended to the appointment at the IMSS' facility and agreed to participate in the study, 157 (11.4\%) refused to participate, and 26 (1.9\%) were hospitalized. Thus, in COSFOMA 2019 a total of 1678 older adults were enrolled. In wave six, all participants were invited to answer a specific module on sleep disorders (ie, insomnia, risk of OSA, daytime sleepiness, and sleep duration). Therefore, the present study includes 1643 (97.9\%) older adults who had complete information on the variables of interest, and $35(2.1 \%)$ were excluded due to incomplete data (Figure 1). This study was carried out according to the Declaration of Helsinki. All participants were informed of the procedures 


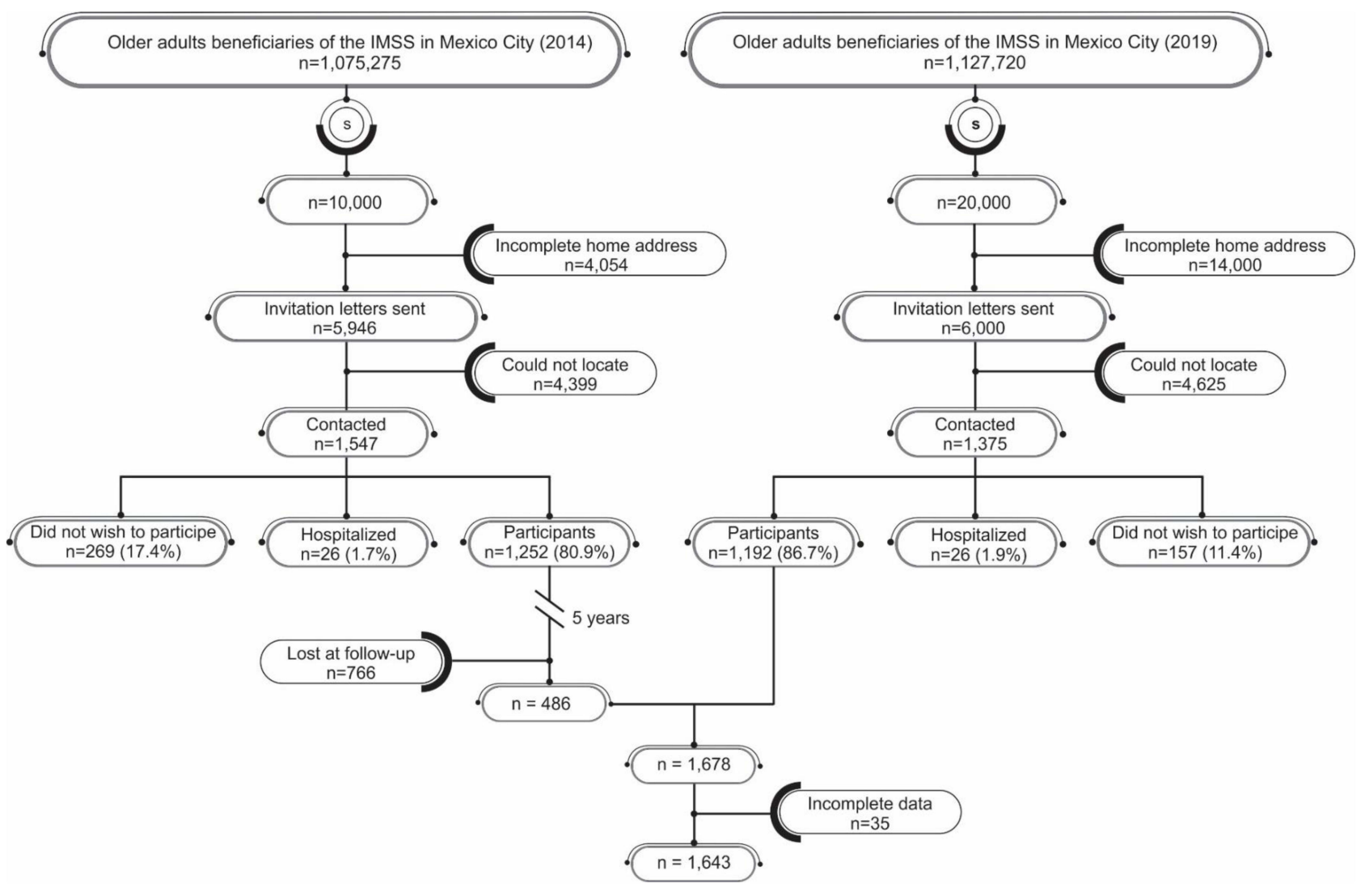

Figure I Enrolment flow chart for the sample. The cohort of obesity, sarcopenia and frailty of older Mexican adults.

Abbreviation: IMSS, Mexican Institute of Social Security.

and signed an informed consent form. COSFOMA and this study were approved by the IMSS National Commission for Scientific Research (Registration number 2012-785067 and R-2018-785-023, respectively).

\section{Measures}

\section{Obstructive Sleep Apnea}

To identify participants with a prior diagnosis of OSA, they responded the following question: "Have you ever been diagnosed with sleep apnea by a physician?" From the number of participants who answered "no", risk of OSA was evaluated through the Berlin Questionnaire. ${ }^{21}$ The Berlin Questionnaire consists of ten questions organized into three categories in which the presence, frequency and intensity of snoring are investigated, as well as the presence of respiratory pauses during sleep (category 1); self-report of non-restorative sleep, fatigue during wakefulness or somnolence while driving (category 2 ) and the presence of obesity or hypertension (category 3). Obesity was determined by calculating the body mass index (BMI) from the measurement of weight and height with a value $\geq 30 \mathrm{~kg} / \mathrm{m} 2$. Hypertension was considered when the older adult reported having a previous medical diagnosis. Having high risk of OSA was considered if the participant presented at least two of the three categories.

\section{Insomnia}

Insomnia was assessed with the Athens Insomnia Scale (AIS). ${ }^{22}$ The AIS considers the diagnostic criteria for insomnia of the 10th Classification of Mental and Behavioral Disorders. The AIS consists of 8 questions that explore the perception of difficulty in inducing sleep, waking up during the night, waking up early in the morning, total sleep time, general quality of sleep, feeling of wellness, general functioning and somnolence during the day. Each question on the AIS is scored from 0 to 3 , where 0 equals no problem and 3 equals a very serious problem. Participants with a score of 6 or more on the AIS (range 0-24) were considered to have insomnia. ${ }^{22}$

\section{Sleepiness}

Sleepiness was assessed with the Epworth Sleepiness Scale, which includes eight questions to assess the 
perception of difficulty staying awake during wakefulness. Participants were asked to evaluate the possibility of falling asleep in eight everyday situations: reading, watching television, sitting in a public place, feeling the need to lie down to rest, sitting and talking to someone, sitting, after eating and as a passenger in a vehicle. Each question was scored from 0 to 3 , where 0 equals to low probability of falling asleep and 3 to high probability of falling asleep. The total score ranges from 0 to 24 . Values greater than 10 were considered sleepiness. $^{23}$

\section{Self-Assessed Sleep Duration}

Sleep duration was measured through self-report with the question: "During the past month, how many hours of actual sleep did you get at night?" Participants' responses were classified into 5 groups according to the National Sleep Foundation ${ }^{24}$ criteria: $<5$ h, From 5 to $<7$ h, From 7 to $<9 \mathrm{~h}$, or $\geq 9 \mathrm{~h}$.

\section{Co-Morbid Insomnia and OSA (COMISA)}

Since insomnia and OSA are two common sleep disorders which may overlap, and both are linked to health adverse outcomes, ${ }^{25}$ we additionally considered co-morbid insomnia and OSA (COMISA) in this study. The identification of co-morbid insomnia in subjects with a prior diagnosis of OSA or at high risk of OSA was based on the presence of insomnia according to the AIS $\geq 6$ and at least one out of three nocturnal symptoms rated as very serious (3) which were: sleep induction, awakenings during the night, and final awakening earlier than desired. Then, a categorical variable was defined as follows: 1) No insomnia/no OSA, 2) Insomnia-alone, 3) OSA-alone, and 4) Co-morbid insomnia and OSA.

\section{Frailty}

The frailty phenotype was evaluated using the proposal by Fried et $\mathrm{al}^{3}$ that entails the evaluation of the presence of five clinical indicators: exhaustion (determined based on whether the participants declared that they felt that everything they did was an effort and/or felt like they could not go on any further), low strength (indicated by grip strength, measured with a dynamometer and adjusted for BMI and sex), involuntary weight loss (considered present when the participant responded having lost $4.5 \mathrm{~kg}$ or more of body weight in the last year), slow walking speed (determined by the time it took the participant to walk a $4.5 \mathrm{~m}$ course, adjusting for sex and height) and low physical activity (assessed by the Physical Activity Scale for the Elderly ${ }^{26}$ ). Each participant was assigned a point for each indicator present and the scores were added to create a variable that took values from 0 to 5 . Older adults with a score $\geq 3$ were defined with frailty as the outcome for the statistical analysis.

\section{Covariates}

Sociodemographic variables such as age (years), sex (men, women), education ( $<6$ years, 6 years, $\geq 7$ years), marital status (currently united; not united) were included. The presence of comorbidities was evaluated by asking the interviewees if they had a medical diagnose of any of the following conditions: diabetes, peptic ulcer, hypothyroidism, arthritis, musculoskeletal diseases, depression, chronic lung disease, peripheral vascular disease, heart disease, cancer, kidney failure, and chronic liver disease. With this information, a categorical variable was defined as follows: none, one, and two or more diseases. Since the definition of the risk of OSA includes hypertension and obesity, we did not use them to indicate comorbidities. The Center for Epidemiologic Studies Depression ScaleRevised (CESD-R) was used to detect the presence of clinically significant depression symptoms using a cut-off of $\leq 57$ points. $^{27}$ Cognitive function was determined with the Mini-Mental State Examination (MMSE), which assesses performance in different cognitive components, such as orientation, attention, immediate and short-term memory, language, and the ability to follow orders. Older adults with a score of 23 or less on the MMSE were considered with cognitive decline. ${ }^{28}$ Nap habit was defined as taking regular naps. Current medication use in relation to drugs for the nervous system that included analgesics, antiepileptics, antiparkinsonism, psycholeptic and psychoanaleptic was incorporated for analysis, apart from comorbidities because often subjects cannot specify diagnosis that motivates its use and they directly affect sleep and frailty. In addition, older adults were asked about current alcohol consumption (yes; no) and tobacco use (never, former smoker, current smoker).

\section{Statistical Analysis}

Descriptive statistics were used to summarize the characteristics of the older adults. Comparisons between frailty groups were carried out using Pearson's chi-square test for categorical variables and Mann-Whitney test for continuous variables. Logistic regression was used to identify the independent association of risk of OSA, insomnia, sleepiness, and sleep duration with frailty, for the entire sample and stratified by sex. Unadjusted and adjusted models 
were performed controlling for age, education, marital status, napping, current alcohol consumption, tobacco use, clinically significant depression symptoms, cognitive decline, comorbidities, and consumption of medications for the nervous system. For the association between COMISA and frailty unadjusted and adjusted logistic regression models were performed only for the entire sample; stratified models by sex were not feasible given the small sample sizes. Multicollinearity was checked using the variance inflation factor and it was not found to be a problem $(\mathrm{VIF}<10)$. Crude and adjusted odds ratios (OR) with 95\% confidence intervals (CI) are reported. All statistical analysis were performed with the Stata 16.1 statistical software package (StataCorp, 2020).

\section{Results}

The average age was $67.1 \pm 5.9$ years. It was found that $10.5 \%(\mathrm{n}=172)$ had frailty and that women were more likely to present it compared to men $(12.7 \%$ vs $8.3 \%, \mathrm{p}=$ $0.003)$. Of the participants, $2.9 \%(n=47)$ self-reported prior diagnosis of OSA. The prevalence of prior diagnosis of OSA was higher in men compared to women $(4.1 \%$ vs $1.6 \%, \mathrm{p}=0.002)$. The percentage of older adults who presented high risk of OSA was $26.1 \%(n=429)$ and there was no significant difference between men and women $(26.5 \%$ vs $25.7 \%, \mathrm{p}=0.726)$. Of the total participants, 30.3\% $(\mathrm{n}=497)$ had insomnia; this sleep disorder was higher in women than in men $(33.2 \%$ vs $27.3 \%$, $\mathrm{p}=0.01)$. Sleepiness was present in $18.9 \%(\mathrm{n}=310)$ of the participants, without observing a statistically significant difference between men and women $(18.6 \%$ vs $19.2 \%, \mathrm{p}=0.773)$.

Table 1 shows the distribution of the characteristics of men and women with or without frailty. In both men and women, participants with frailty were older, less educated, had a higher percentage of clinically significant depression symptoms and cognitive decline, a lower proportion of alcohol consumption, and a higher percentage of comorbidities than older adults in the non-frail group. Use of medications related to nervous system was more frequent in frail women than in non-frail $(33.7 \%$ vs $16.9 \%$, p < 0.001 ) but not in men. Regarding sleep disorders, the report of insomnia was significantly higher in older adults with frailty in men $(47.1 \%$ vs $25.6 \%, \mathrm{p}<0.001)$ and in women $(54.8 \%$ vs $30.0 \%, \mathrm{p}<0.001)$. The percentage of somnolent men was higher among frail than non-frail $(33.8 \%$ vs $17.2 \%, p=0.001)$. It seems that the percentage of high risk of OSA was higher among frail men than non- frail men but there was no statistical difference $(35.3 \%$ vs $25.7 \%, p=0.08$ ). In contrast, frail women were more likely to have high risk of OSA compared to non-frail women, and a higher reported sleepiness, although this was not significant. Sleep duration and the report of naps were not different according to frailty condition, neither in men nor in women.

Table 2 shows the results of the logistic regression models for the association between sleep disorders and frailty. In the entire study sample, unadjusted and adjusted model show that having prior diagnosis of OSA was not associated with frailty. In contrast, the unadjusted model shows that compared to older adults without risk of OSA, people with risk of OSA were $76 \%$ more likely to present frailty (95\% CI: 1.26, 2.46). This association remained significant in the model adjusted for sex, age, education, marital status, insomnia, sleepiness, sleep duration, napping, current alcohol consumption, tobacco use, clinically significant depression symptoms, cognitive decline, comorbidities, and use of nervous system medications $(\mathrm{OR}=1.59,95 \% \mathrm{CI}: 1.07,2.38)$. The presence of insomnia increased the odds of frailty in the unadjusted $(\mathrm{OR}=2.79, \quad 95 \%$ CI: 2.03, 3.85) and adjusted $(\mathrm{OR}=2.11,95 \% \mathrm{CI}: 1.41,3.16)$ logistic regression analysis. In the unadjusted model, sleepiness was significantly associated with a greater possibility of frailty $(\mathrm{OR}=1.91$, 95\% CI: 1.34, 2.72); however, when controlling for the covariates, the association did not maintain statistical significance $(\mathrm{OR}=1.38,95 \% \mathrm{CI}$ : 0.90, 2.12). Self-assessed sleep duration was not associated with frailty.

Regarding the stratified analysis by sex, neither having prior diagnosis of OSA nor risk of OSA were associated with frailty in men (Table 2). Having insomnia significantly increased the chances of frailty both in the unadjusted $(\mathrm{OR}=2.59 ; 95 \% \mathrm{CI}: 1.56,4.28)$ and adjusted models $(\mathrm{OR}=1.88 ; 95 \% \mathrm{CI}: 1.01,3.52)$. Table 2 shows sleepiness as a possible variable related to frailty $(\mathrm{OR}=.46,95 \% \mathrm{CI}: 1.44,4.20)$ but after the adjusted analysis, the association lost statistical significance $(\mathrm{OR}=1.51,95 \% \mathrm{CI}: 0.79,2.88)$. Regarding selfassessed sleep duration, no association with frailty was observed in any model.

In the case of women, participants with prior diagnosis of OSA were significantly more likely to have frailty (unadjusted OR $=3.90 ; 95 \% \mathrm{CI}: 1.17$, 13.03). Although no significant association was seen when the model was controlled for covariates $(\mathrm{OR}=4.02$; 95\% CI: 0.99 , 16.22). It was observed that the participants with risk of 
Table I Characteristics of the 1643 Older Adults According to Frailty and Sex

\begin{tabular}{|c|c|c|c|c|c|c|c|}
\hline \multirow[t]{4}{*}{ Variables } & \multirow{4}{*}{$\begin{array}{l}\text { Total } \\
n=\mid 643\end{array}$} & \multicolumn{3}{|l|}{ Men } & \multicolumn{3}{|l|}{ Women } \\
\hline & & \multicolumn{3}{|l|}{$n=823$} & \multicolumn{3}{|l|}{$n=820$} \\
\hline & & $\begin{array}{l}\text { Without } \\
\text { Frailty }\end{array}$ & Frailty & $\mathbf{p}$ & $\begin{array}{l}\text { Without } \\
\text { Frailty }\end{array}$ & Frailty & $\mathbf{p}$ \\
\hline & & $n=755$ & $n=68$ & & $n=716$ & $n=104$ & \\
\hline Age (years) & $67.1 \pm 5.9$ & $67.1 \pm 5.7$ & $69.9 \pm 8.7$ & 0.033 & $66.1 \pm 4.9$ & $71.6 \pm 7.9$ & $<0.001$ \\
\hline \multicolumn{8}{|l|}{ Education (n, \%) } \\
\hline$<6$ years & III (6.8) & $36(4.8)$ & $6(8.8)$ & $<0.001$ & $45(6.3)$ & $24(23.1)$ & $<0.001$ \\
\hline 6 years & $295(18.0)$ & $124(16.4)$ & $25(36.8)$ & & $126(17.6)$ & $20(19.2)$ & \\
\hline$\geq 7$ years & $1237(75.3)$ & $595(78.8)$ & $37(54.4)$ & & $545(76.1)$ & $60(57.7)$ & \\
\hline Married/civil union (n, \%) & $1004(61.1)$ & $601(79.6)$ & $53(77.9)$ & 0.745 & $314(43.9)$ & $36(34.6)$ & 0.075 \\
\hline \multicolumn{8}{|l|}{ OSA (n, \%) } \\
\hline Prior diagnosis of OSA & $47(2.9)$ & $33(4.4)$ & I (I.5) & 0.180 & $9(1.3)$ & $4(3.9)$ & 0.001 \\
\hline High risk of OSA & $429(26.1)$ & $194(25.7)$ & $24(35.3)$ & & $172(24.0)$ & $39(37.5)$ & \\
\hline Without OSA & $1167(71.0)$ & $528(69.9)$ & $43(63.2)$ & & $535(74.7)$ & $61(58.7)$ & \\
\hline Insomnia (n, \%) & $497(30.3)$ & $193(25.6)$ & $32(47.1)$ & $<0.001$ & $215(30.0)$ & $57(54.8)$ & $<0.001$ \\
\hline Sleepiness (n, \%) & $310(18.9)$ & $130(17.2)$ & $23(33.8)$ & 0.001 & $130(18.2)$ & $27(26.0)$ & 0.059 \\
\hline \multicolumn{8}{|l|}{ Self-assessed sleep duration ( $\mathrm{n}, \%)$} \\
\hline$<5 h$ & $255(15.5)$ & $114(15.1)$ & $13(19.1)$ & 0.356 & $109(15.2)$ & $19(18.3)$ & 0.395 \\
\hline $5-6 \mathrm{~h}$ & $795(48.4)$ & $374(49.5)$ & $38(55.9)$ & & $339(47.4)$ & $44(42.3)$ & \\
\hline $7-8 \mathrm{~h}$ & $534(32.5)$ & $244(32.3)$ & $15(22.1)$ & & $24 I(33.7)$ & $34(32.7)$ & \\
\hline$\geq 9 \mathrm{~h}$ & $59(3.6)$ & $23(3.1)$ & $2(2.9)$ & & $27(3.8)$ & $7(6.7)$ & \\
\hline Napping (n, \%) & $595(36.2)$ & $308(40.8)$ & $30(44.1)$ & 0.594 & $221(30.9)$ & $36(34.6)$ & 0.441 \\
\hline Current alcohol consumption (n, \%) & $594(34.9)$ & $347(46.0)$ & $14(20.6)$ & $<0.001$ & $200(27.9)$ & $13(12.50)$ & 0.001 \\
\hline \multicolumn{8}{|l|}{ Tobacco consumption (n, \%) } \\
\hline Never & $851(51.8)$ & $294(38.9)$ & $31(45.6)$ & 0.528 & $457(63.8)$ & $69(66.3)$ & 0.575 \\
\hline Former smoker & $489(29.8)$ & $285(37.8)$ & $24(35.3)$ & & $156(21.8)$ & $24(23.1)$ & \\
\hline Current smoker & $303(18.4)$ & $176(23.3)$ & $13(19.1)$ & & $103(14.4)$ & II (10.6) & \\
\hline $\begin{array}{l}\text { Clinically significant depression symptoms } \\
(\mathrm{n}, \%)\end{array}$ & $124(7.5)$ & $36(4.8)$ & $16(23.5)$ & $<0.001$ & $44(6.2)$ & $28(26.9)$ & $<0.001$ \\
\hline Cognitive decline (n, \%) & $379(23.1)$ & $159(21.1)$ & $29(42.7)$ & $<0.001$ & $148(20.7)$ & $43(4 I .4)$ & $<0.001$ \\
\hline \multicolumn{8}{|l|}{ Comorbidities (n, \%) } \\
\hline None & $829(50.5)$ & 407 (53.9) & $26(38.2)$ & 0.019 & $353(49.3)$ & $43(41.4)$ & 0.069 \\
\hline One & $587(35.7)$ & $269(35.6)$ & $29(42.7)$ & & $253(35.3)$ & $36(34.6)$ & \\
\hline Two or more & $227(13.8)$ & $79(10.5)$ & $13(19.1)$ & & $110(15.4)$ & $25(24.0)$ & \\
\hline $\begin{array}{l}\text { Use of medications related to nervous system } \\
(\mathrm{n}, \%)\end{array}$ & $272(16.6)$ & $102(13.5)$ & $14(20.6)$ & 0.108 & $121(16.9)$ & $35(33.7)$ & $<0.001$ \\
\hline
\end{tabular}

Abbreviation: OSA, obstructive sleep apnea.

OSA were more likely to be frail in the unadjusted $(\mathrm{OR}=1.99 ; 95 \% \mathrm{CI}: 1.29,3.08)$ and in the adjusted model $(\mathrm{OR}=1.84 ; 95 \% \mathrm{CI}: 1.05,3.22)$ compared to women with no OSA. Women with insomnia faced greater chances of frailty (adjusted OR: 2.38; 95\% CI: 1.35, 4.20) compared to women without insomnia. Neither sleepiness nor self-assessed sleep duration was associated with frailty in women. 
Table 2 Logistic Regression for the Association Between Sleep Disorders and Frailty, Stratified by Sex

\begin{tabular}{|c|c|c|c|}
\hline \multicolumn{2}{|c|}{ Variables } & $\begin{array}{l}\text { Unadjusted } \\
\text { OR }(95 \% \mathrm{Cl})\end{array}$ & $\begin{array}{l}\text { Adjusted }^{\mathrm{a}} \\
\text { OR }(95 \% \mathrm{Cl})\end{array}$ \\
\hline \multirow[t]{4}{*}{ All } & $\begin{array}{l}\text { OSA } \\
\text { Without OSA } \\
\text { Prior diagnosis of OSA } \\
\text { High risk of OSA }\end{array}$ & $\begin{array}{l}1.00 \\
1.22(0.47,3.14) \\
1.76(1.26,2.46)^{* *}\end{array}$ & $\begin{array}{l}1.00 \\
1.23(0.43,3.50) \\
1.59(1.07,2.38)^{*}\end{array}$ \\
\hline & Insomnia & $2.79(2.03,3.85)^{* * *}$ & $2.11(1.4 I, 3.16)^{* * *}$ \\
\hline & Sleepiness & $1.91(1.34,2.72)^{* * *}$ & $1.38(0.90,2.12)$ \\
\hline & $\begin{array}{l}\text { Self-assessed sleep duration } \\
<5 \mathrm{~h} \\
5-6 \mathrm{~h} \\
7-8 \mathrm{~h} \\
\geq 9 \mathrm{~h}\end{array}$ & $\begin{array}{l}1.42(0.89,2.28) \\
1.14(0.78,1.65) \\
1.00 \\
I .78(0.83,3.84)\end{array}$ & $\begin{array}{l}0.65(0.36,1.18) \\
0.96(0.63,1.47) \\
1.00 \\
1.42(0.60,3.38)\end{array}$ \\
\hline \multirow[t]{4}{*}{ Men } & $\begin{array}{l}\text { OSA } \\
\text { Without OSA } \\
\text { Prior diagnosis of OSA } \\
\text { High risk of OSA }\end{array}$ & $\begin{array}{l}1.00 \\
0.37(0.05,2.79) \\
1.52(0.90,2.57)\end{array}$ & $\begin{array}{l}1.00 \\
0.32(0.04,2.65) \\
1.19(0.65,2.19)\end{array}$ \\
\hline & Insomnia & $2.59(1.56,4.28)^{* * *}$ & $1.88(1.01,3.52)^{*}$ \\
\hline & Sleepiness & $2.46(I .44,4.20)^{* *}$ & I.5I $(0.79,2.88)$ \\
\hline & $\begin{array}{l}\text { Self-assessed sleep duration } \\
\quad<5 \mathrm{~h} \\
5-6 \mathrm{~h} \\
7-8 \mathrm{~h} \\
\geq 9 \mathrm{~h}\end{array}$ & $\begin{array}{l}1.85(0.85,4.03) \\
I .65(0.89,3.07) \\
1.00 \\
I .41(0.30,6.57)\end{array}$ & $\begin{array}{l}1.10(0.42,2.84) \\
1.62(0.82,3.24) \\
1.00 \\
1.29(0.24,6.85)\end{array}$ \\
\hline \multirow[t]{4}{*}{ Women } & $\begin{array}{l}\text { OSA } \\
\text { Without OSA } \\
\text { Prior diagnosis of OSA } \\
\text { High risk of OSA }\end{array}$ & $\begin{array}{l}1.00 \\
3.90(1.17,13.03)^{*} \\
1.99(1.29,3.08)^{* *}\end{array}$ & $\begin{array}{l}1.00 \\
4.02(0.99,16.22) \\
1.84(1.05,3.22)^{*}\end{array}$ \\
\hline & Insomnia & $2.83(1.86,4.29)^{* * *}$ & $2.38(1.35,4.20)^{* *}$ \\
\hline & Sleepiness & $1.58(0.98,2.55)$ & $\mathrm{I} .4 \mathrm{I}(0.77,2.6 \mathrm{I})$ \\
\hline & $\begin{array}{l}\text { Self-assessed sleep duration } \\
<5 \mathrm{~h} \\
5-6 \mathrm{~h} \\
7-8 \mathrm{~h} \\
\geq 9 \mathrm{~h}\end{array}$ & $\begin{array}{l}1.24(0.67,2.26) \\
0.92(0.57,1.48) \\
1.00 \\
1.84(0.74,4.55)\end{array}$ & $\begin{array}{l}0.49(0.22,1.11) \\
0.64(0.35,1.15) \\
1.00 \\
1.42(0.48,4.19)\end{array}$ \\
\hline
\end{tabular}

Notes: ${ }^{a}$ Adjusted for age, education, married/civil union, napping, current alcohol consumption, current smoking, clinically significant depression symptoms, cognitive decline, comorbidities, use of medications related to nervous system.*** $p<0.001,{ }^{* *} p<0.01,{ }^{*} p<0.05$.

Abbreviations: OSA, obstructive sleep apnea; OR, odds ratio; $\mathrm{Cl}$, confidence interval.

Table 3 shows that COMISA was more frequent among frail than non-frail participants. Besides, the results of the logistic regression models to investigate the association of COMISA with frailty showed that compared to participants with no insomnia and no OSA, insomnia alone, OSA alone, and co-morbid insomnia and OSA were associated significantly with frailty in the unadjusted model. After controlling for covariates, results showed that insomnia-alone $(\mathrm{OR}=$ 2.00, 95\% CI: 1.23, 3.26), OSA-alone (OR $=2.02$, $95 \%$ CI 1.29, 3.18), and co-morbid insomnia and OSA $(\mathrm{OR}=6.46,95 \% \mathrm{CI}: 2.37,17.58)$ significantly increased 
Table 3 Frailty According to COMISA in Older Adults

\begin{tabular}{|c|c|c|c|c|c|c|}
\hline \multirow[t]{2}{*}{ Variables } & Total & Without Frailty & Frailty & \multirow[t]{2}{*}{$\mathbf{p}$} & Unadjusted & Adjusted $^{\mathrm{a}}$ \\
\hline & $n=1643$ & $n=|47|$ & $n=172$ & & OR $(95 \% \mathrm{Cl})$ & OR $(95 \% \mathrm{Cl})$ \\
\hline \multicolumn{7}{|l|}{ COMISA (n, \%) } \\
\hline No insomnia/ no OSA & $872(53.1)$ & $813(55.3)$ & $59(34.3)$ & $<0.001$ & 1.00 & 1.00 \\
\hline Insomnia-alone & $295(18.0)$ & $250(17.0)$ & $45(26.2)$ & & $2.48(1.64,3.75)^{* * *}$ & $2.00(1.23,3.26)^{* *}$ \\
\hline OSA-alone ${ }^{b}$ & $447(27.2)$ & $389(26.4)$ & $58(33.7)$ & & $2.05(\mathrm{I} .40,3.0 \mathrm{I})^{* * * *}$ & $2.02(1.29,3.18)^{* *}$ \\
\hline Co-morbid insomnia and OSA ${ }^{\mathrm{b}}$ & $29(1.8)$ & $19(1.3)$ & $10(5.8)$ & & $7.25(3.23,16.30)^{* * *}$ & $6.46(2.37,17.58)^{* * *}$ \\
\hline
\end{tabular}

Notes: ${ }^{a}$ Logistic regression model adjusted for age, education, married/civil union, self-assessed sleep duration, sleepiness, napping, current alcohol consumption, current smoking, clinically significant depression symptoms, cognitive decline, comorbidities, and use of medications related to nervous system. ${ }^{b}$ Includes prior diagnosis of OSA or high risk of OSA measured with the Berlin questionnaire. *** $p<0.001,{ }^{* *} p<0.01$.

Abbreviations: COMISA, co-morbid insomnia and obstructive sleep apnea; OSA, obstructive sleep apnea; OR, odds ratio; $\mathrm{Cl}$, confidence interval.

the odds of frailty, compared to those without no insomnia and no OSA.

\section{Discussion}

This study documents that after adjustment for sociodemographic and health characteristics, risk of OSA, and insomnia were associated with frailty in older adults. Having a prior diagnosis of OSA was not associated with frailty neither globally nor sex-disaggregated. In stratified analyses by sex, risk of OSA was found to be independently associated with frailty only in women; and insomnia was associated with frailty in men and women. Globally, the association between sleepiness and frailty was attenuated and did not remain statistically significant in the adjusted model. Sleepiness was associated with frailty only in men in the unadjusted model and was no longer significant in the final model. No association was observed between self-assessed sleep duration and frailty, globally or stratified by sex.

The results that emerge from this research concur with other studies that have shown a relationship between sleep and frailty. ${ }^{10}$ Although it is still not entirely conclusive, some previous research suggests that the association of sleep complaints, sleep quality, and insomnia on frailty may be differentiated according to sex. ${ }^{6,15,29}$

In the particular case of sleep breathing disorders, Endeshaw et al reported that the apnea-hypopnea index $>30$ significantly increased the chances of being frail only in the group of women and not in the group of men. ${ }^{14}$ In a cross-sectional study made up of men, Ensrud et al showed that an index of respiratory events (RDI $>15$ ) was associated with frailty. ${ }^{19}$ However, after studying the same cohort of men for 3.4 years, Ensrud et al observed that OSA did not increase the incidence of frailty, but it did increase the risk of death and the only respiratory disorder related to frailty and death was hypoxemia during sleep defined with $10 \%$ of the recording time with saturation less than $90 \% .{ }^{18}$ On the other hand, in a cohort of women, Spira et al found no effect between the apnea-hypopnea index and the incidence of difficulty in moving (a criterion that may be similar to the walking speed of frailty). ${ }^{30}$ Because there are few investigations that have addressed the relationship between OSA (either measured with a questionnaire or with polysomnography) and frailty, ${ }^{14,18,19}$ it is important that other studies resume this issue and confirm the phenomenon, as well as the pathophysiological mechanisms involved in these apparent differences between men and women.

The association between insomnia and frailty has scarcely been explored with non-conclusive findings. A previous cross-sectional study in this cohort had also reported sex differences with respect to the association between insomnia and frailty, showing that insomnia is an independent factor for frailty in women. ${ }^{29}$ However, it missed to consider other co-occurring sleep disturbances such as risk of OSA or daytime sleepiness. In the study by Vaz Fragoso et al where insomnia was defined based on the insomnia severity index, an association with frailty was found to loses statistical significance when adjusting for comorbidities and use of medications. ${ }^{16}$ A recent study that investigated the association between insomnia symptoms and frailty found that sleep-onset insomnia but no sleep-maintenance explained frailty through reduced physical performance. ${ }^{31}$ In our study, the association between insomnia and frailty was significant globally and in women even in the model adjusted for comorbidities and medication use; the existence of an ethnic or cultural factor that explains this discrepancy cannot be ruled out and further studies are required in this regard. 
As diseases are not mutually exclusive in real life, insomnia co-exists with OSA in a group of patients, ${ }^{25}$ thus, we assessed its association with frailty. First, results revealed that the prevalence of co-morbid insomnia and risk of OSA in older adults was $1.8 \%$. As far as we know, there is no report of COMISA in older adults but this value is similar to the prevalence reported in an Australian population-based study which found $1.5 \%$ of adults with COMISA evaluated at disorder level. ${ }^{32}$ Second, in line to what we found analyzing the risk of OSA and insomnia as independent variables, when we took into account COMISA, we found that older adults who faced OSAalone, insomnia alone or co-morbid insomnia and OSA had greater chances of frailty than older adults with no such sleep disorders. The association between COMISA and frailty, although imprecise due to small sample size, appears to be more than the simple additive effect of each factor and this finding suggests that COMISA is relevant not only to clinical settings but also in the general population. ${ }^{32}$ However, further studies to assess this association are warranted.

The fact that insomnia, risk of OSA and COMISA explained frailty may be expected as they have been related to hyperactivity of the adrenocortical axis. OSA is characterized by repeated interruptions of breathing during sleep, with consequent nocturnal hypoxemia and sleep fragmentation. Apneas markedly disrupt Phase 3 of sleep and REM sleep. ${ }^{33}$ Since it has been suggested that hypoxia could be a pathway that promotes sympathetic activation, it could be thought that such activation could promote oxidative stress ${ }^{34}$ which has been previously related with frailty. ${ }^{35}$ For their part, sleep fragmentation and insomnia promote a state of hypervigilance that is also associated with an increase in sympathetic activity and the adrenocortical axis $^{36}$ derived from which the levels of inflammatory molecules such as interleukin-6 and C-reactive protein as well as catabolic processes, increase the deterioration of the organism, affect the immune system that would lead to frailty in older adults. ${ }^{37}$ Given that prevalence of insomnia and risk of OSA in this study sample were high and being disorders for which there is an effective pharmacological and non-pharmacological treatment, screening of older adults should be part of a routine health assessment in this age group.

Despite the fact that men had higher prevalence of prior diagnosis of OSA than women, our data showed that after controlling for covariates, there was no independent association between prior diagnosis of OSA with frailty both in men and women. Unfortunately, we did not collect details regarding patients' treatment to explain the lack of significant relationship between prior diagnosis of OSA and frailty. Since participants of this study are beneficiaries of the Mexican Institute of Social Security, a mandatory social security system in Mexico, they may potentially be undergoing CPAP treatment for sleep apnea which may result in improving cardiovascular and metabolic outcomes. Future research should carefully examine whether CPAP treatment truly improves frailty in older people with OSA.

Now, understanding the reason why we observe the association between high risk of OSA and frailty in women and not in men represents a challenge due to the fact that the percentage of risk of OSA observed in this research was not different between men and women when OSA typically has been considered a more frequent sleep disorder in the male population than in the female population. ${ }^{38}$ Although it seems that this is also reflected in older people, ${ }^{14}$ the prospective evidence regarding the incidence of OSA suggests that is distinct from that occurs in middle-aged people. A longitudinal study by Tishler et al showed that the risk of 5-year incidence of OSA increased modestly with age in men and rose steadily and markedly in women. However, the authors did not find evidence to support the idea that the discrepancy in the incidence of OSA differed by sex after age 50 years. ${ }^{39}$ According to studies that have focused on comparisons of OSA in women through the lifecycle have found that after menopause the decline in levels of sexual hormones may explain the development of OSA. ${ }^{40}$ At the moment, we do not know the mechanism that could explain that the association between OSA and frailty is enhanced in women, but we suppose that it could be partly due to body composition. Older adult women tend to be more obese, which means a pro-inflammatory state that also promotes the collapse of the upper airway during sleep. Although in our work we did not have inflammatory markers to confirm this, we observed that frail women had a significantly greater neck circumference than non-frail women (35.7 \pm $2.7 \mathrm{~cm}$ vs $35.0 \pm 2.6 \mathrm{~cm}, \mathrm{p}<0.05)$. However, such difference was not seen in men $(40.0 \pm 2.9 \mathrm{~cm}$ vs $39.9 \pm$ $3.4, \mathrm{p}=0.915)$. In addition, women are also more susceptible to the effects of sarcopenia. ${ }^{41}$ Sarcopenia has been linked to OSA since it could be a marker of the presence of low muscle quality, essential to carry out ventilatory efforts and maintain patency of the pharynx. ${ }^{42}$ On the other hand, the decrease in the levels of sexual hormones 
could be partly responsible for the propensity to collapse of the posterior oropharynx and promote OSA. ${ }^{38,43}$ It cannot be ruled out that other reasons to explain the nonsignificant relationship between high risk of OSA and frailty in male participants in this study include the low accessibility of diagnostic, and OSA and frailty susceptibility. Even though men typically are more likely to be diagnosed or treated with OSA than women, men usually have low use of health care. In addition, men are more susceptible to the effects of OSA on mortality compared to women, especially in men aged $40-70$ years. ${ }^{44}$ Evidence has shown that as a result of complex interrelationship between psychosocial, structural, and behavioral factors, women have higher prevalence of frailty and survival rates than men. ${ }^{45}$ These features may reflect a survival bias against men with high risk of OSA.

In this study, we observed a significant association between sleepiness and frailty in the unadjusted statistical analysis for the whole sample and in men, but it was lost in the adjusted analysis. This differs from the work of Vaz Fragoso et al who found a strong association between sleepiness and frailty. ${ }^{16}$ This discrepancy, despite the use of the same instrument and cut-off point to define sleepiness, could be due to older age, schooling and a higher percentage of women in the study by Vaz Fragoso; additionally, the study by Vaz Fragoso et al did not include any OSA assessment tool. In contrast, Ensrud et al reported, for a male cohort, that both in the cross-sectional and longitudinal analysis, the association between daytime sleepiness and frailty is lost in the adjusted statistical models, while the perception of poor sleep quality as well as nocturnal hypoxemia increased the risk of frailty or death. ${ }^{18,19}$ Given the discordant results, more studies are required on the relation between excessive sleepiness and frailty.

Previous research has documented that sleeping less or more hours than recommended is related to frailty. However, we observed that self-assessed sleep duration was not associated with frailty. The association between sleep duration and frailty is not entirely conclusive and the discrepancy could be due to the fact that few studies have considered other sleep characteristics such as the presence of sleep disorders or indicators of sleep quality. For example, in the longitudinal study conducted by Guida et al, it was observed that sleep duration was not associated with frailty or mortality, and instead sleep fragmentation, nocturnal awakenings, as well as a lower total percentage sleep conferred an effect to develop frailty or dying. ${ }^{13}$

This research has some limitations. First, the design is cross-sectional so it is susceptible to reverse causality.
Second, we evaluated the risk of OSA through self-report using the Berlin Questionnaire. Although the Berlin Questionnaire is a standardized tool, it provides a sensitivity of $0.76,0.77$, and 0.88 for mild, moderate and severe OSA, respectively; and its specificity is $0.59,0.44$, and 0.38 for mild, moderate and severe OSA, respectively. ${ }^{46}$ Future studies should take this limitation into account and use overnight polysomnography or home sleep apnea test to objectively confirm OSA. The use of a questionnaire to assess insomnia and sleepiness can also be questioned because they are not usually complete tests for clinical diagnosis. However, the Berlin Questionnaire, the Athens Insomnia Scale and the Epworth Scale for sleepiness have been previously used or validated in the Mexican population. ${ }^{22,47,48}$ As strengths, our study includes a representative sample of older adults in a community-based cohort in Mexico City, with a good representation of both sexes, where validated questionnaires of common clinical use were collected directly by trained personnel.

\section{Conclusion}

Overall, the results indicate that risk of OSA is independently associated with frailty in women. Insomnia is associated in both men and women. In addition, this study suggests that COMISA is associated with frailty in older people. We hope that results that document the apparent discrepancy according to sex, motivate future studies to try to confirm it and try to clarify better the ways in which this occurs. Besides, this important distinction regarding sex difference should be taken into consideration in the clinical evaluation of older adults. Prospective studies on sleep and frailty are still lacking, thus, additional work is needed to investigate if these sleep disturbances may predict the onset of frailty. From the point of view of disease prevention, these results suggest a need for strategies aimed at improving sleep hygiene and detecting patients with risk of OSA and insomnia.

\section{Ethics Approval and Consent to Participate}

This study was carried out according to the Declaration of Helsinki. All participants were informed of the research procedures and signed a letter of consent prior to participating. Study protocol were approved by the IMSS National Research Committee (Registration No. 2012785-067 and R-2018-785-023). 


\section{Funding}

This study was supported by a grant from the Fund for the Promotion of Health Research (FIS/IMSS/PROT/G18/ 1813), IMSS.

\section{Disclosure}

The authors report no conflicts of interest in this work.

\section{References}

1. Collard RM, Boter H, Schoevers RA, Oude Voshaar RC. Prevalence of frailty in community-dwelling older persons: a systematic review. $J$ Am Geriatr Soc. 2012;60(8):1487-1492. doi:10.1111/j.15325415.2012.04054.x

2. Siriwardhana DD, Hardoon S, Rait G, Weerasinghe MC, Walters KR. Prevalence of frailty and prefrailty among community-dwelling older adults in low-income and middle-income countries: a systematic review and meta-analysis. BMJ Open. 2018;8:e018195. doi:10.1136/ bmjopen-2017-018195

3. Fried LP, Tangen CM, Walston J, et al. Frailty in older adults: evidence for a phenotype. J Gerontol a Biol Sci Med Sci. 2001;56 (3):M146-M156. doi:10.1093/gerona/56.3.M146

4. Morley JE, Vellas B, van Kan GA, et al. Frailty consensus: a call to action. J Am Med Dir Assoc. 2013;14(6):392-397. doi:10.1016/j. jamda.2013.03.022

5. Piovezan R, Poyares D, Tufik S. Frailty and sleep disturbances in the elderly: possible connections and clinical implications. Sleep Sci. 2013;6(4):175-179.

6. Balomenos V, Ntanasi E, Anastasiou CA, et al. Association between sleep disturbances and frailty: evidence from a population-based study. J Am Med Dir Assoc. 2020;22(3):551-558.e1. doi:10.1016/j. jamda.2020.08.012

7. Nakakubo S, Makizako H, Doi T, et al. Long and short sleep duration and physical frailty in community-dwelling older adults. J Nutr Health Aging. 2018;22(9):1066-1071. doi:10.1007/s12603-018-1116-3

8. Akın S, Özer FF, Zararsız GE, et al. The impact of sleep duration on frailty in community-dwelling Turkish older adults. Sleep Biol Rhythms. 2020;18(3):243-248. doi:10.1007/s41105-020-00264-y

9. Baniak LM, Yang K, Choi J, Chasens ER. Long sleep duration is associated with increased frailty risk in older community-dwelling adults. J Aging Health. 2018;32(1):42-51. doi:10.1177/0898264318803470

10. Wai JLT, Yu DSF. The relationship between sleep-wake disturbances and frailty among older adults: a systematic review. J Adv Nurs. 2020;76(1):96-108. doi:10.1111/jan.14231

11. Kang I, Kim S, Kim BS, Yoo J, Kim M, Won CW. Sleep latency in men and sleep duration in women can be frailty markers in community-dwelling older adults: the Korean frailty and aging cohort study (KFACS). $J$ Nutr Health Aging. 2019;23(1):63-67. doi:10.1007/s12603-018-1109-2

12. Del Brutto O, Mera R, Sedler J, et al. The effect of age in the association between frailty and poor sleep quality: a population-based study in community-dwellers (The Atahualpa Project). J Am Med Dir Assoc. 2016;17(3):269-271. doi:10.1016/j. jamda.2015.12.009

13. Guida JL, Alfini AJ, Gallicchio L, Spira AP, Caporaso NE, Green PA. Association of objectively measured sleep with frailty and 5-year mortality in community-dwelling older adults. Sleep. 2021;44(7): zsab003. doi:10.1093/sleep/zsab003

14. Endeshaw YW, Unruh ML, Kutner M, Newman AB, Bliwise DL. Sleep-disordered breathing and frailty in the cardiovascular health study cohort. Am J Epidemiol. 2009;170(2):193-202. doi:10.1093/ aje/kwp108
15. Moreno-Tamayo K, Manrique-Espinoza B, Rosas-Carrasco O, PérezMoreno A, Salinas-Rodríguez A. Sleep complaints are associated with frailty in Mexican older adults in a rural setting. Geriatr Gerontol Int. 2017;17(12):2573-2578. doi:10.1111/ggi.13111

16. Vaz Fragoso CA, Gahbauer EA, Van Ness PH, Gill TM. Sleep-wake disturbances and frailty in community-living older persons. $\mathrm{J} \mathrm{Am}$ Geriatr Soc. 2009;57(11):2094-2100. doi:10.1111/j.15325415.2009.02522.x

17. Mallampalli MP, Carter CL. Exploring sex and gender differences in sleep health: a society for women's health research report. $J$ Women's Heal. 2014;23:553-562. doi:10.1089/jwh.2014.4816

18. Ensrud KE, Blackwell T, Ancoli-Israel S, et al. Sleep disturbances and risk of frailty and mortality in older men. Sleep Med. 2012;13 (10):1217. doi:10.1016/j.sleep.2012.04.010

19. Ensrud KE, Blackwell TL, Redline S, et al. Sleep disturbances and frailty status in older community-dwelling men. J Am Geriatr Soc. 2009;57(11):2085-2093. doi:10.1111/j.1532-5415.2009.02490.x

20. Sánchez-García S, García-Peña C, Salvà A, et al. Frailty in community-dwelling older adults: association with adverse outcomes. Clin Interv Aging. 2017;12:1003-1011. doi:10.2147/CIA. $\mathrm{S} 139860$

21. Netzer NC, Stoohs RA, Netzer CM, Clark K, Strohl KP. Using the Berlin Questionnaire to identify patients at risk for the sleep apnea syndrome. Ann Intern Med. 1999;131(7):485-491. doi:10.7326/00034819-131-7-199910050-00002

22. Nenclares A, Jiménez-Genchi A. Estudio de la validación de la traducción al español de la escala Atenas de insomnio. Salud Ment. 2005;28:34-39.

23. Johns MW. A new method for measuring daytime sleepiness: the Epworth sleepiness scale. Sleep. 1991;14(6):540-545. doi:10.1093/ sleep/14.6.540

24. Hirshkowitz M, Whiton K, Albert SM, et al. National sleep foundation's sleep time duration recommendations: methodology and results summary. Sleep Heal. 2015;1(1):40-43. doi:10.1016/j.sleh.2014.12.010

25. Sweetman A, Lack L, Bastien C. Co-morbid insomnia and sleep apnea (COMISA): prevalence, consequences, methodological considerations, and recent randomized controlled trials. Brain Sci. 2019;9 (12):371. doi:10.3390/brainsci9120371

26. Washburn RA, McAuley E, Katula J, Mihalko SL, Boileau RA. The physical activity scale for the elderly (PASE): evidence for validity. $J$ Clin Epidemiol. 1999;52(7):643-651. doi:10.1016/S0895-4356(99) 00049-9

27. Sánchez-García S, Juárez-Cedillo T, Gallegos-Carrillo K, Gallo JJ, Wagner FA, García-Peña C. Frecuencia de los síntomas depresivos entre adultos mayores de la Ciudad de México [Frequency of depressive symptoms among older adults in Mexico City]. Salud Ment. 2012;35(1):71-77. Spanish.

28. Reyes De-beaman S, Beaman PE, Garcia-Peña C, et al. Validation of a modified version of the mini-mental state examination (MMSE) in Spanish. Aging Neuropsychol Cogn. 2004;11(1):1-11. doi:10.1076/ anec.11.1.1.29366

29. Moreno-Tamayo K, Manrique-Espinoza B, Ortiz-Barrios LB, Á C-B, Ramírez-García E, Sánchez-García S. Insomnia, low sleep quality, and sleeping little are associated with frailty in Mexican women. Maturitas. 2020;136:7-12. doi:10.1016/j.maturitas.2020.03.005

30. Spira AP, Stone KL, Rebok GW, et al. Sleep-disordered breathing and functional decline in older women. J Am Geriatr Soc. 2014;62 (11):2040-2046. doi:10.1111/jgs.13108

31. Tang JY, Luo H, Tse M, Lum TY, Wong GH, Li SX. The relationship between insomnia symptoms and frailty in community-dwelling older persons: a path analysis. Sleep Med. 2021;84:237-243. doi:10.1016/j. sleep.2021.05.039

32. Sweetman A, Melaku YA, Lack L, et al. Prevalence and associations of co-morbid insomnia and sleep apnoea in an Australian population-based sample. Sleep Med. 2021;82:9-17. doi:10.1016/j. sleep.2021.03.023 
33. Ratnavadivel R, Chau N, Stadler D, Yeo A, McEvoy RD, Catcheside PG. Marked reduction in obstructive sleep apnea severity in slow wave sleep. J Clin Sleep Med. 2009;5(6):519-524. doi: $10.5664 /$ jesm. 27651

34. Johansson P, Svensson E, Alehagen U, Jaarsma T, Broström A. The contribution of hypoxia to the association between sleep apnoea, insomnia, and cardiovascular mortality in community-dwelling elderly with and without cardiovascular disease. Eur J Cardiovasc Nurs. 2015;14(3):222-231. doi:10.1177/1474515114524072

35. Soysal P, Isik AT, Carvalho AF, et al. Oxidative stress and frailty: a systematic review and synthesis of the best evidence. Maturitas. 2017;99:66-72. doi:10.1016/j.maturitas.2017.01.006

36. Bonnet MH, Arand DL. Hyperarousal and insomnia: state of the science. Sleep Med Rev. 2010;14(1):9-15. doi:10.1016/j.smrv.2009.05.002

37. Isik AT, Veronese N, Prina AM, et al. Inflammation and frailty in the elderly: a systematic review and meta-analysis. Ageing Res Rev. 2017;31:1-8.

38. Lin CM, Davidson TM, Ancoli-Israel S. Gender differences in obstructive sleep apnea and treatment implications. Sleep Med Rev. 2008;12(6):481-496. doi:10.1016/j.smrv.2007.11.003

39. Tishler PV, Larkin EK, Schluchter MD, Redline S. Incidence of sleep-disordered breathing in an urban adult population: the relative importance of risk factors in the development of sleep-disordered breathing. JAMA. 2003;289(17):2230-2237. doi:10.1001/jama.28 9.17.2230

40. Huang T, Lin BM, Redline S, Curhan GC, Hu FB, Tworoger SS. Type of menopause, age at menopause, and risk of developing obstructive sleep apnea in postmenopausal women. Am J Epidemiol. 2018;187(7):1370-1379. doi:10.1093/aje/kwy011
41. Batsis JA, Mackenzie TA, Barre LK, Lopez-Jimenez F, Bartels SJ. Sarcopenia, sarcopenic obesity and mortality in older adults: results from the national health and nutrition examination survey III. Eur J Clin Nutr. 2014;68(9):1001-1007. doi:10.1038/ejcn.2014.117

42. Morley JE, Sanford A, Bourey R. Sleep apnea: a geriatric syndrome. J Am Med Dir Assoc. 2017;18(11):899-904. doi:10.1016/j.jamda.2017.08.020

43. Wimms A, Woehrle H, Ketheeswaran S, Ramanan D, Armitstead J. Obstructive sleep apnea in women: specific issues and interventions. Biomed Res Int. 2016;2016:1764837. doi:10.1155/2016/1764837

44. Punjabi NM, Caffo BS, Goodwin JL, et al. Sleep-disordered breathing and mortality: a prospective cohort study. PLoS Med. 2009;6(8): e1000132. doi:10.1371/journal.pmed.1000132.

45. Kane AE, Howlett SE. Sex differences in frailty: comparisons between humans and preclinical models. Mech Ageing Dev. 2021;198:111546. doi:10.1016/j.mad.2021.111546.

46. Chiu H-Y, Chen P-Y, Chuang L-P, et al. Diagnostic accuracy of the Berlin questionnaire, STOP-BANG, STOP, and Epworth sleepiness scale in detecting obstructive sleep apnea: a bivariate meta-analysis. Sleep Med Rev. 2017;36:57-70. doi:10.1016/j.smrv.2016.10.004

47. Sandoval-Rincón M, Alcalá-Lozano R, Herrera-Jiménez I, JiménezGenchi A. Validación de la escala de somnolencia de Epworth en población mexicana. Gac Med Mex. 2013;149(4):409-416. doi:10.1590/S0124-00642007000400008

48. Guerrero-Zúñiga S, Gaona-Pineda EB, Cuevas-Nasu L, et al. Prevalencia de síntomas de sueño y riesgo de apnea obstructiva del sueño en México. Salud Publica Mex. 2018;60:347-355. doi:10.21149/9280
Nature and Science of Sleep

\section{Publish your work in this journal}

Nature and Science of Sleep is an international, peer-reviewed, open access journal covering all aspects of sleep science and sleep medicine, including the neurophysiology and functions of sleep, the genetics of sleep, sleep and society, biological rhythms, dreaming, sleep disorders and therapy, and strategies to optimize healthy sleep.
The manuscript management system is completely online and includes a very quick and fair peer-review system, which is all easy to use. Visit http://www.dovepress.com/testimonials.php to read real quotes from published authors. 